\title{
Was sagen die Leitlinien?
}

\author{
Unsere Leserbefragung hat gezeigt: Sie wünschen, dass das Thema Leitlinien auch in der MMW \\ stärkere Berücksichtigung findet. Wir greifen diese Anregung gerne auf und starten mit einem \\ neuen Modul. Dabei erscheint es nicht sinnvoll, die Leitlinien einfach schematisch „herunterzu- \\ beten". Vielmehr möchten wir an Einzelbeispielen versuchen, eine evidenzbasierte Lösung zu \\ finden. Wir laden auch Sie ein, eigene Patienten vorzustellen, bei denen sich die Frage stellt: \\ Was sagen die Leitlinien?
}

\section{Helicobacter-Eradikation: Auch bei funktioneller Dyspepsie?}

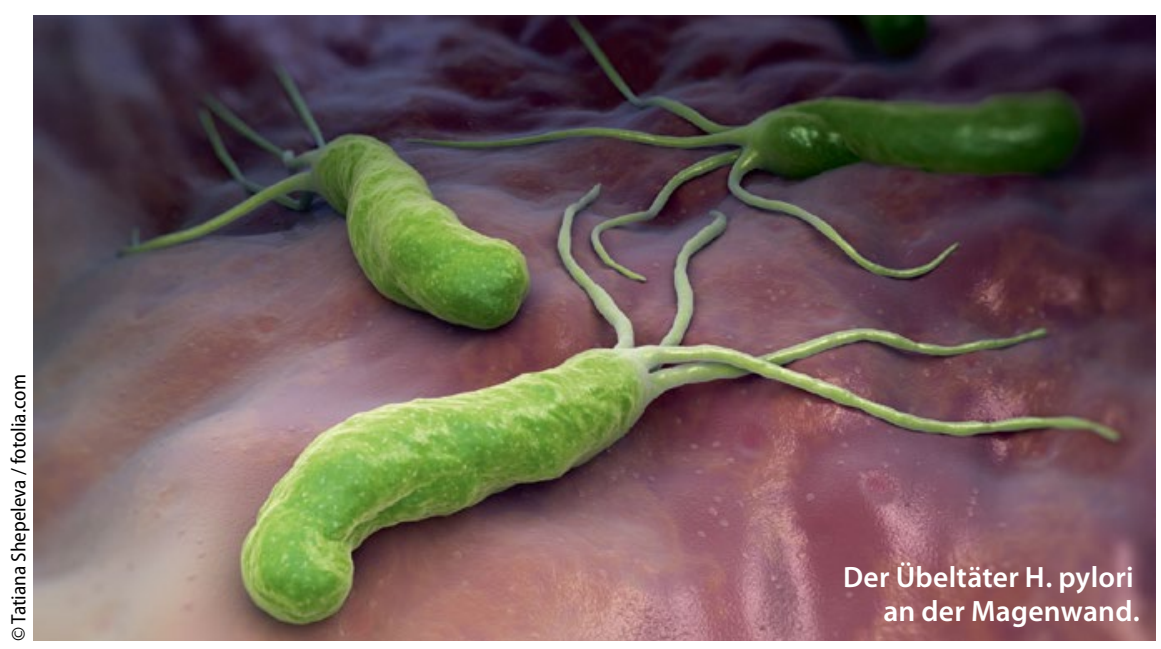

?

Ein 32-jähriger, bisher gesunder Patient klagt über einen anhaltenden Druck in der Magengegend, vor allem postprandial ohne Alarmsymptome wie Gewichtsabnahme oder Anämie. Die probatorische Gabe eines PPI bringt keine Besserung. Bei der anschließend durchgeführten Gastroskopie wird eine H.p.-Infektion mittels Urease-Schnell-Test nachgewiesen. Der Gastroenterologe empfiehlt jetzt die Eradikation. Ist das leitliniengerecht? Die Helicobacter-pylori-Infektion bei einem peptischen Ulcus ventriculi oder duodeni ist eine obligate $\mathrm{Be}$ handlungsindikation mit dem Ziel der Keimeradikation (Empfehlungsgrad A, Evidenzstärke 1a). Doch die Eradikation bei einer länger bestehenden Non-ulcerDyspepsie mit negativem endoskopischen Befund führt nach vorliegenden Studienergebnissen nur bei 5-10\% der Patienten zu einer anhaltenden Besserung. Trotzdem gilt auch hier der Empfehlungsgrad A, allerdings nur mit der Evidenzstärke $1 b$, zumal die Wahrscheinlichkeit relevanter gastrointestinaler Nebenwirkungen nur bei $10-25 \%$ liegt. Somit handelt es sich um eine fakultative Behandlungsindikation, wobei die Entscheidung individuell in Abhängigkeit von der Dauer und Intensität der Beschwerden und evtl. auch demWunsch des Patienten entsprechend getroffen werden sollte.

- http://www.dgvs.de/fileadmin/user_upload/Leitlinien/Helicobacter_pylori/Leitlinie_Helicobacter.pdf

\section{Kommentar}

\section{DAS LEID MIT DEN LEITLINIEN}

Das Thema "Leitlinien“ ist in der Medizin omnipräsent. Bei jeder Diskussion bzw. Fortbildung wird früher oder später die Frage gestellt: Was sagen die Leitlinien dazu? Auch für Sie als Hausarzt gilt, dass Sie sich bei der Diagnostik und Therapie an den Leitlinien orientieren sollen, nicht nur, aber auch aus haftungsrechtlichen Gründen; denn sollte es zu einer juristischen Auseinandersetzung kommen, wird der Richter immer die Frage an den Gutachter richten: Was sagen die Leitlinien?

Doch gerade in der Hausarztpraxis stößt man mit der Forderung nach Einhaltung der Leitlinien oft an Grenzen; zum einen gibt es für viele alltägliche Probleme überhaupt keine Leitlinien, hier ist Ihre Intuition und Empirie unverzichtbar. Zum anderen sind Leitlinien nur für klar definierte Krankheitsbilder formuliert. Dürfen, können oder müssen diese aber auch uneingeschränkt für ältere multimorbide Patienten gelten? Wenn Sie einen älteren Patienten mit fünf verschiedenen Erkrankungen leitliniengerecht behandeln, so haben Sie zumindest die "Chance", inn mit dieser Polypharmazie umzubringen. Dieses Zitat eines Pharmakologen mag überspitzt klingen, aber ganz falsch ist es nicht. Auch sollte man immer wieder daran erinnern, dass Leitlinien nur als Leitplanken fungieren, diese also eine gewisse Bandbreite an Möglichkeiten erlauben. Auch ein Abweichen von den offiziellen Empfehlungen ist nicht nur möglich, sondern sogar geboten, aber man muss es begründen können und sollte es auch dokumentieren.

Dr. Peter Stiefelhagen 